\title{
DEMONSTRATING THE FEASIBILITY OF MULTIMODAL NEUROIMAGING DATA CAPTURE WITH A WEARABLE ELECTOENCEPHALOGRAPHY + FUNCTIONAL NEAR- INFRARED SPECTROSCOPY (EEG+FNIRS) IN SITU
}

\author{
Dybvik, Henrikke; \\ Erichsen, Christian Kuster; \\ Steinert, Martin \\ Norwegian University of Science and Technology
}

\begin{abstract}
We developed a wearable experimental sensor setup featuring multimodal EEG+fNIRS neuroimaging applicable for in situ experiments of human behavior in interaction with technology. A low-cost electroencephalography (EEG) was integrated with a wearable functional Near-Infrared Spectroscopy (fNIRS) system, which we present in two parts. Paper A provide an exhaustive description of setup infrastructure, data synchronization process, a procedure for usage, including sensor application, and ensuring high signal quality. This paper (Paper B) demonstrate the setup's usability in three distinct use cases: a conventional human-computer interaction experiment, an in situ driving experiment where participants drive a car in the city and on the highway, and an ashtanga vinyasa yoga practice in situ. Data on cognitive load from highly ecologically valid experimental setups are presented, and we discuss lessons learned. These include acceptable and unacceptable artefacts, data quality, and constructs possible to investigate with the setup.
\end{abstract}

Keywords: EEG+fNIRS, in situ experiments, Human behaviour in design, User centred design, Research methodologies and methods

\section{Contact:}

Dybvik, Henrikke

Norwegian University of Science and Technology

Department of Mechanical and Industrial Engineering

Norway

henrikke.dybvik@ntnu.no

Cite this article: Dybvik, H., Erichsen, C. K., Steinert, M. (2021) 'Demonstrating the Feasibility of Multimodal Neuroimaging Data Capture with a Wearable Electoencephalography + Functional Near-Infrared Spectroscopy (EEG+FNIRS) in Situ', in Proceedings of the International Conference on Engineering Design (ICED21), Gothenburg, Sweden, 16-20 August 2021. DOI:10.1017/pds.2021.90 


\section{INTRODUCTION}

Engineering design research is trending towards increasing experiments that integrate physiology and neuroimaging measurements (Balters \& Steinert, 2017; Goucher-Lambert et al., 2019; Hay et al., 2020; Steinert \& Jablokow, 2013). Such experiments often exist with a trade-off between experimental control and ecological validity (Hay et al., 2020), which is problematic since laboratory settings simply cannot provide results replicable in the real word (Cairns \& Cox, 2008; Okamoto et al., 2004). Highly ecologically valid (aka in situ) studies demonstrate how humans appropriate technological solutions in their intended context, accommodating the often unpredictable, real-world environments in which technology is used (Consolvo et al., 2007). Thus, they are suited for design research (Balters \& Steinert, 2017; Hay et al., 2020; Mayseless et al., 2019). Electroencephalography (EEG) and functional near-infrared spectroscopy (fNIRS) are two portable and complementing neuroimaging modalities serving as a substitute for neuroscience gold standard fMRI, by measuring electrical brain activity and cerebral hemodynamic response respectively (Herold et al., 2018; Jacko, 2012; Pinti et al., 2018). However, portable neuroimaging systems are expensive, often come at the cost of low resolution and limited options (Ahn \& Jun, 2017; Cisler et al., 2019; Piper et al., 2014). Moreover, most neuroimaging studies are often confined to the comfort of a laboratory or an educational setting, and not in situ (Gero \& Milovanovic, 2020; Goucher-Lambert et al., 2019; Hay et al., 2020; Steinert \& Jablokow, 2013). Thus, there is a need for a portable experimental sensor-setup featuring multimodal neuroimaging data capture at a lower financial threshold, and there is a need to demonstrate its feasibility in situ.

\subsection{The study goal}

This work contributes by demonstrating the feasibility of concurrent EEG and fNIRS measurements in situ. We increase ecological validity within these demonstrations by testing real-world experimental use cases of increasing complexity. This paper demonstrates three use cases: a conventional humancomputer interaction experiment, a driving experiment in situ involving city and highway driving, and a moving yoga practice in situ. Together with concreate design experiment examples this exemplification expands the range of what is possible within a design research experiment.

The paper briefly describes sensor placement, pre-processing and analysis of EEG and fNIRS, before demonstrating the cases in section 3. Here we provide a description of experimental procedure, results and lessons learned for each case. Section 4 provide examples of potential design experiments. Concluding remarks follow.

\section{SENSOR PLACEMENT, DATA PROCESSING AND ANALYSIS}

Sensor placement. We used a wearable EEG+fNIRS (Dybvik et al., Under Review). FNIRS optodes were placed over the prefrontal cortex (montage by NIRx Medical Technologies, LLC) using 8 sources and 7 detectors. The sources (denoted Sx) were placed as follows: S1: F3, S2: AF7, S3: AF3, S4: Fz, S5: Fpz, S6: AF4, S7: F4, and S8: AF8. The detectors (denoted Dx) were placed as follows D1: F5, D2: F1, D3: Fp1, D4: AFz, D5: F2, D6: Fp2, and D7: F6. This configuration gives 40 channels. EEG electrodes were placed as follows: AFp1, AFp2, C3, C4, P7, P8, O1 and O2, and one reference electrode placed on each earlobe. Optodes and electrodes were positioned according to the five percent system (Oostenveld \& Praamstra, 2001).

Data processing and analysis. For EEG, an initial power spectral density analysis within the frequency bands delta $(1-4 \mathrm{~Hz})$, theta $(4-8 \mathrm{~Hz})$, alpha $(8-13 \mathrm{~Hz})$, beta $(13-30 \mathrm{~Hz})$, and gamma $(30-100$ $\mathrm{Hz}$ ) was performed, a common approach for cognitive load investigations (Borghini et al., 2014). Preprocessing included bandpass filtering $(1-50 \mathrm{~Hz})$, and a notch filter $(48-52 \mathrm{~Hz})$ to attenuate line noise, using a Hamming windowed sinc FIR filter. Bad channels and artifacts were removed by visual inspection. Power frequency density was calculated at $1 \mathrm{~Hz}$ using Welch's power spectral density estimate. Results were converted to $\mu V^{2} / \mathrm{Hz}$, calculating total band power and relative band power. For fNIRS, pre-processing included conversion from raw data to optical density, then conversion to hemoglobin concentration using the modified Beer-Lambert Law. Then a general linear model regression, using an autoregressive pre-whitening method with iteratively reweighted least-squares (AR-IRLS) (Barker et al., 2013; Santosa et al., 2018), was performed for first-level statistics. This procedure is more robust to effects of physiology and motion compared to prior recommendations 
(e.g. bandpass filtering, motion artifact correction, cardiac response removal etc.), and is therefore now recommended (Santosa et al., 2018). EEGLAB (Delorme \& Makeig, 2004) and NIRS toolbox (Santosa et al., 2018) in MATLAB was used.

\section{DEMONSTRATION CASES}

This section presents concrete test cases selected to demonstrate flexibility in our multimodal EEG+fNIRS sensor setup, through a variety of experimental scenarios. These include a conventional human-computer interaction setup, in situ city and highway driving, and in situ ashtanga vinyasa yoga practice. We describe each respective case, show resulting data, and detail lessons learned. Procedure for sensor application is described in-depth elsewhere (Dybvik et al., Under Review). Collected data was processed according to the pipeline described in section 2 .

\subsection{Case 1: Conventional laptop setup}

\subsubsection{Description of conventional human-computer interaction setup}

A conventional computer setup was used as a pilot experiment to test signal quality and analyze data. Participants were tasked with playing a computer game at different difficulties and responding to random auditory alarms using an Arduino device. Computer game difficulty was set to easy in the first condition, and hard in the second condition. The participant was first exposed to a baseline condition, sitting and relaxing in front of a static screen with instructions to relax for two minutes. An assimilation period followed, before exposure to each condition for four minutes, with a two-minute resting period between conditions. This experiment was set up in a standard office cleared for everything except desks, chairs, and a wall separating participant and experimenter, see Fig. 1a.

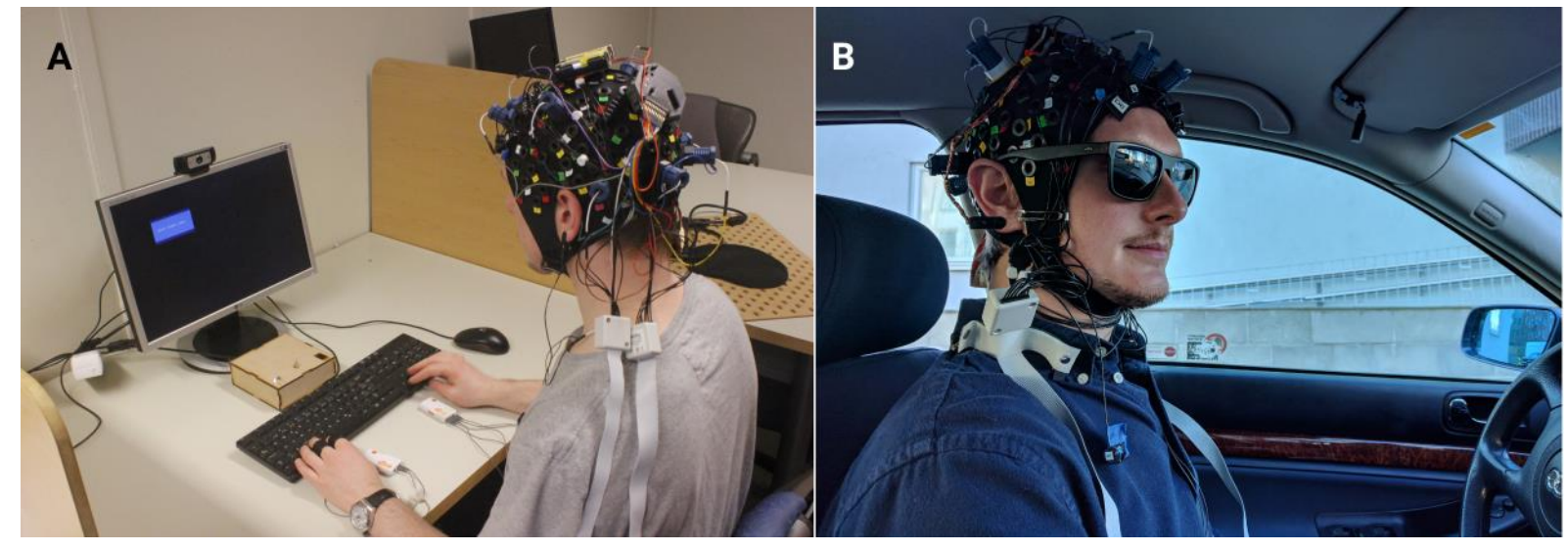

Figure 1. Full sensor setup in A) a conventional laptop configuration, and B) in situ driving.

\subsubsection{Results from preliminary data analysis}

Result of preliminary analysis EEG. The power spectral density (PSD) plots indicated decent signal quality during baseline, and easy condition (Fig. 2a. and 2b). The PSD plot for condition hard indicated a moderate amount of inference from the fNIRS, expressed by regular peaks at harmonics of the fNIRS sampling rate $(7.8 \mathrm{~Hz})$ (Fig. 2c). Thus, this data was not included in further analysis. According to literature, increasing cognitive workload correlates with decreased power in the alpha band at central, and posterior locations (Antonenko et al., 2010; Klimesch, 1999; Sterman et al., 1993), and an increase in the theta band at frontal locations (Borghini et al., 2014). The results obtained during this pilot experiment indicate the same trends, showing increased theta power at location AFp2, and alphasuppression in central, and posterior locations $\mathrm{C} 3, \mathrm{C} 4, \mathrm{P} 7, \mathrm{P} 8, \mathrm{O} 1$, and $\mathrm{O} 2$ (see Fig. 3). This indicates that playing the computer game induce higher cognitive load than a baseline condition, as expected.

Result of preliminary analysis fNIRS. Three representative data slices for each condition were selected for illustration in Fig. 4, which plots concentration changes in oxygenated hemoglobin, $\Delta \mathrm{HbO}_{2}$, from all channels. Differing mental demands can be seen in the graph as changes along the yaxis, which allows for visual observation of changes in cognitive load. The scale of the y-axis depends on participant, context and fNIRS calibration, and is not absolute. Fig. 4 clearly shows the cardiac response as periodic peaks at $\sim 1 \mathrm{~Hz}$ throughout the data, and we observe an overall trending line over 
time and condition. Apart from a couple of spikes from slight motion artifacts (at 13 and 15s) this selection is an example of data of good quality. The general trend for this participant is an increased cognitive load during condition hard and a lower demand during condition easy, although not as low as the baseline. We clearly see a difference in neural activation in the three conditions. The participant is working harder mentally during the game with high difficulty.
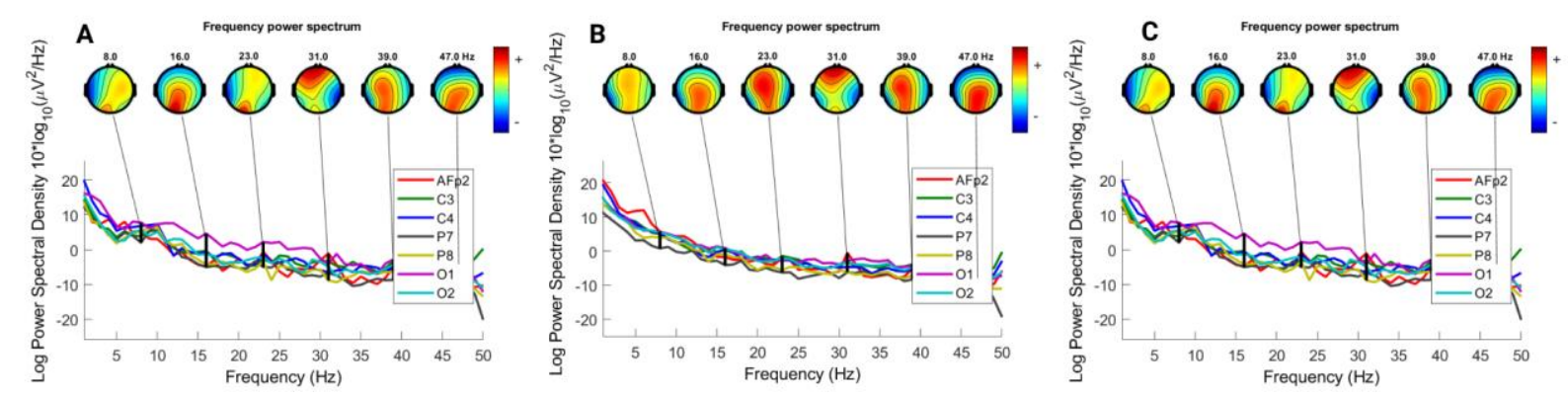

Figure 2. Power spectral density plots for the baseline $(A)$, easy (B), and hard condition (C).
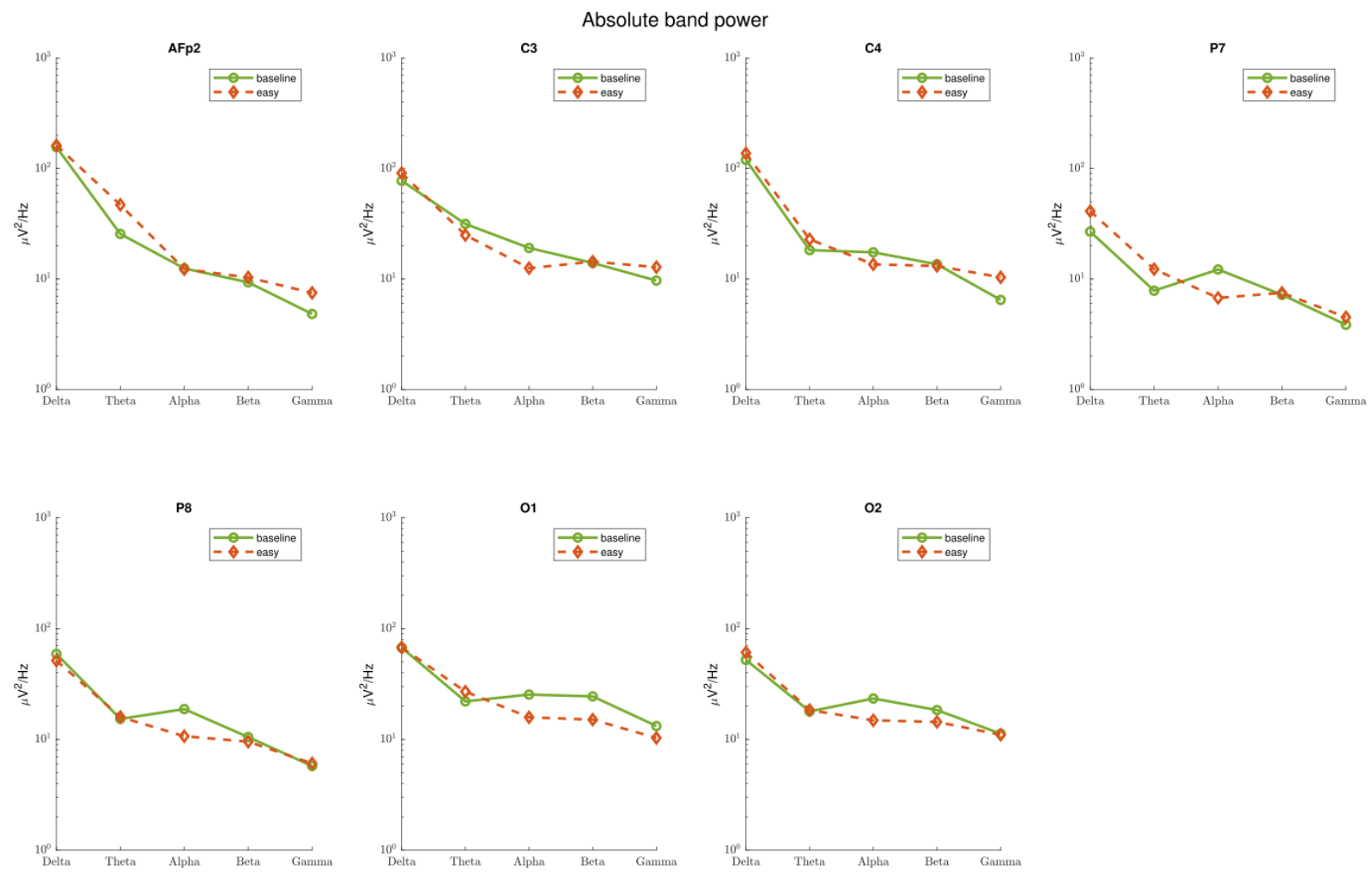

Figure 3. Comparison of spectral band power during baseline and easy condition. One channel was discarded in pre-processing.

\subsubsection{Lessons learned - Identifying bad data}

Equally important as identifying high quality data is the ability to determine what poor data looks.

Identifying bad EEG data. Crosstalk with fNIRS will exhibit peaks in the FFT-plot at harmonics of the fNIRS sampling rate, which can be seen in Fig. 2c, but also in OpenBCI GUI during visual inspection prior to recording. Furthermore, high signal amplitudes $(>50 \mu \mathrm{V})$ might indicate presence of excessive noise in the signal. Very poor electrical contact between electrodes and scalp is indicated by "Near railed" or "Railed" in the time-series plot.

Identifying bad fNIRS data. Bad channels are indicated as red in NIRStar 15.2's Quality Scale tool. If such data is recorded, large, inconsistent signal fluctuations (spikes) in some channels dominate the data as seen in Fig 4b, and they will be discarded in an analysis. Close investigation of Fig. 4b reveal good channels fluctuating around zero, with a visible cardiac response. 

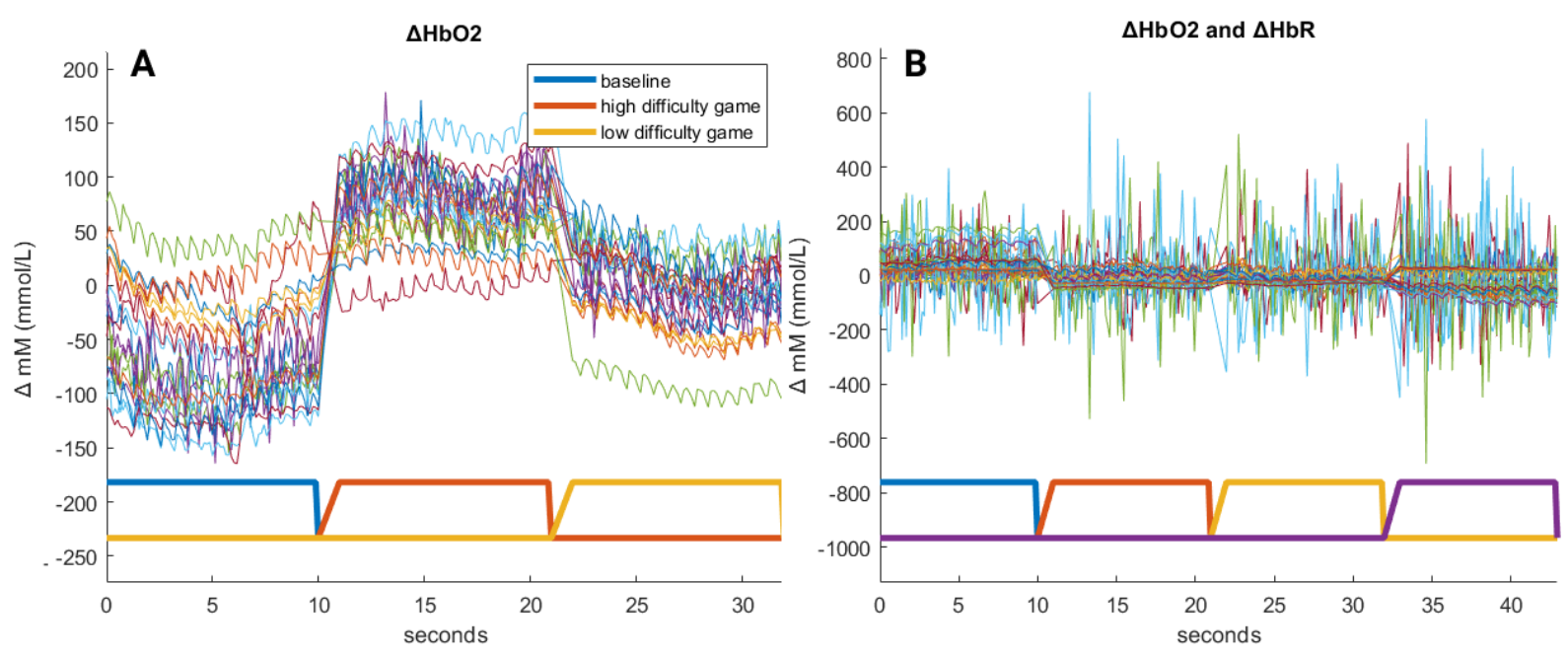

Figure 4. A) $\Delta \mathrm{HbO}_{2}$ over time show increased neural activating during high difficulty.

B) fNIRS data with insufficient quality.

\subsection{Case 2: In situ city and highway driving}

\subsubsection{Description of an in situ driving experiment}

A pilot experiment was conducted to investigate brain activity while driving a car to test the setup's feasibility in in situ environments, and test the ability to differentiate between various levels of cognitive load. This pilot experiment was performed on two separate days, with two participants (one male, one female). Fig. 1b depicts one participant wearing the sensors. Additionally, a video was recorded simultaneously using a web camera. A two-minute baseline was recorded while the participant relaxed inside the parked car. Afterwards, participants were instructed to drive around the city center following vocal directions from the experimenter in the front passenger seat. After 20 minutes, participants were instructed to take an exit to the highway. A 20-minute recording of highway driving was made after crossing city border. We continued data collection afterwards to test the setup's battery life, to benchmark for in situ experiments without access to external power sources. We managed a total of 66 minutes of recorded data, with the laptop battery being the limiting factor. Prior to the experiment we hypothesized higher cognitive load associated with city driving than highway driving due to a more complex environment, and increased handling of the car.

\subsubsection{Results from preliminary data analysis}

Result of preliminary analysis EEG. For visual artifact removal, a five-minute data slice starting at the tenth minute of city and highway, was extracted. The full two minutes of baseline was processed. During visual inspection, $54 \%$ of city, $44 \%$ of highway, and $25 \%$ of baseline data were rejected. Thus, analysis is based on $106 \mathrm{~s}$. of baseline data, $137 \mathrm{~s}$. of city data, and $169 \mathrm{~s}$. of highway data. Increased cognitive load has been associated with increased relative theta-power at frontal regions (Borghini et al., 2014) and decreased alpha-power at parietal and occipital regions (Borghini et al., 2014). The results (Fig. 5) show a relative increase in theta-power in the frontal regions (AFp1, AFp2), in accordance with hypothesized cognitive load: baseline < highway < city. Additionally, we observe an inverse trend within the alpha band at central and posterior locations $(\mathrm{C} 3, \mathrm{C} 4, \mathrm{P} 8, \mathrm{O} 1, \mathrm{O} 2)$, i.e. decreasing alpha-power with increasing cognitive load. There is a substantial difference in relative alpha power between baseline and the two driving conditions. The difference between the two driving conditions is smaller, but the general trend of lower alpha power with hypothesized increased cognitive load holds true for all central and posterior locations, except for $\mathrm{C} 4$. Here, relative alphapower is marginally higher during city driving compared to highway driving. Still, overall results indicate highest cognitive load imposed by city, followed by highway driving, and baseline condition. 

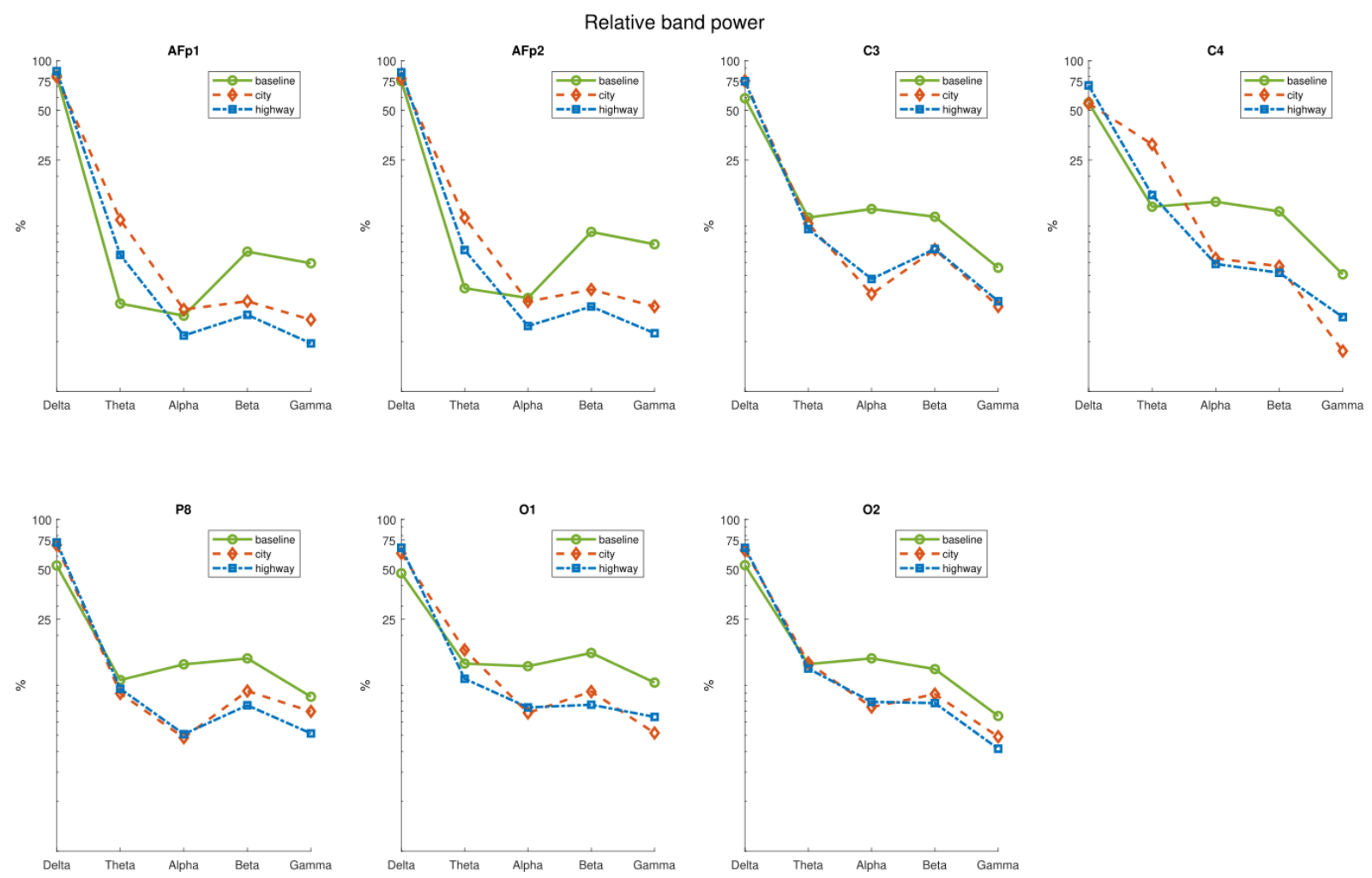

Figure 5. Relative spectral band power. Electrode P7 discarded due to signal quality.

Result of preliminary analysis fNIRS. Three representative data slices were selected for illustrating concentration changes in oxygenated $\left(\Delta \mathrm{HbO}_{2}\right)$ and deoxygenated $(\triangle \mathrm{HbR})$ hemoglobin from three periods during the drive: resting state (baseline), city driving and highway. In Fig. 6a we see $\Delta \mathrm{HbO}_{2}$, which increase with increased brain activation. Fig. $6 \mathrm{~b}$ depicts $\Delta \mathrm{HbR}$, which decrease with brain activation. The optodes' placement over the prefrontal cortex is indicative of cognitive load. As such, we observe an increase in the driver's cognitive demand during city driving compared to baseline and highway. Interestingly highway driving seems to impose less cognitive demand than a resting state (baseline), contradictory to EEG results. In part, this discrepancy may result from different data slices being analyzed, but also from difference in technical principle of EEG and fNIRS. Mental fatigue may be a reason for lower cognitive load during highway compared to baseline (as measured by fNIRS), in which case it supports literature that highlights drivers' mental fatigue as major risk and cause factor for road accidents (Ahn et al., 2016; Ingre et al., 2006; Lal \& Craig, 2001).
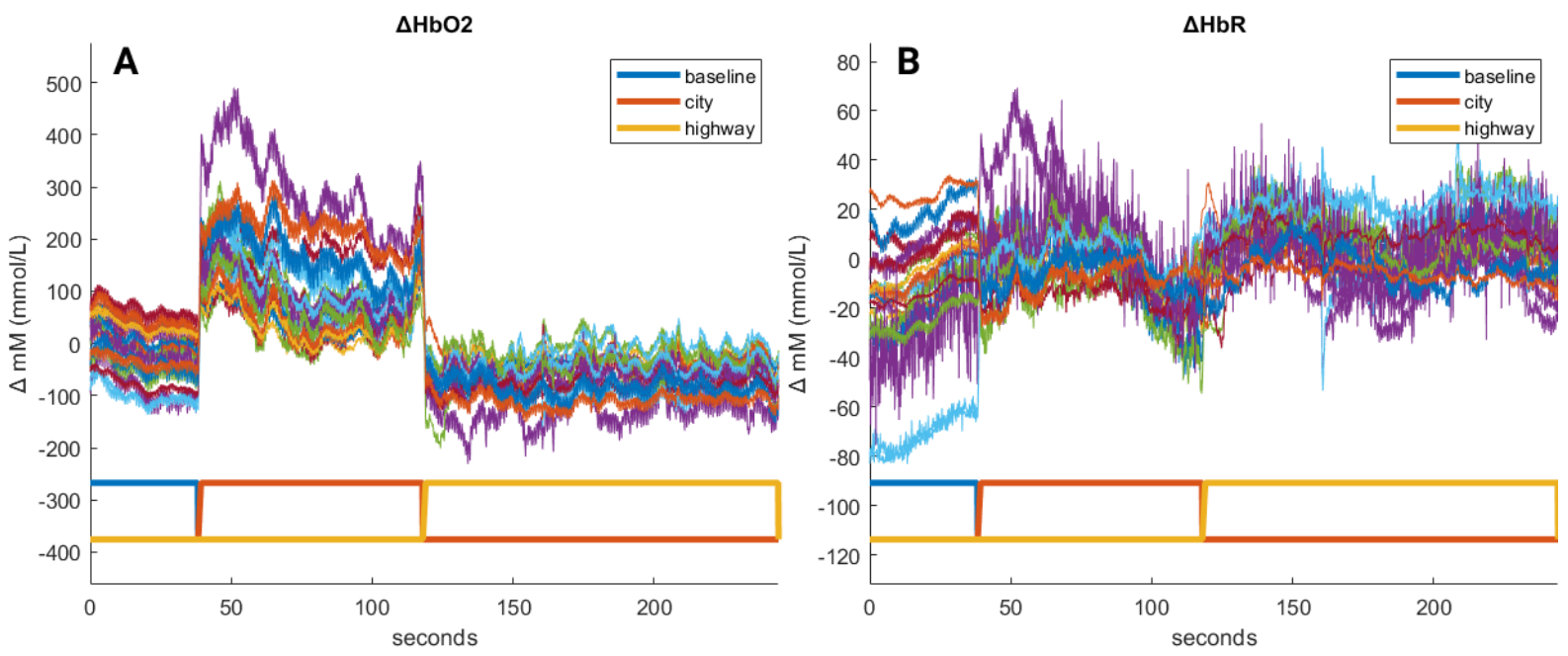

Figure 6. Changes in $\triangle H_{b O_{2}}(A)$ and $\triangle H b R(B) . \Delta H b R$ channels are centered around zero, whereas $\triangle \mathrm{HbO}_{2}$ channels fluctuate more. 


\subsubsection{Lessons learned - The setup enable in situ studies of important research topics}

Changing experimental setup from a stationary classical experiment room to a mobile in situ car driving experiment did not produce major challenges or any corrupt data. In fact, the $50 \mathrm{~Hz}$ interference in EEG from building power lines disappeared. The level of noise did increase (notably motion artifacts from muscle movement, and ocular artifacts). This could be mitigated by marking movements captured by video and removing this data. Furthermore, there exists several methods for artifact removal in EEG-signals that preserve more raw data (Jung et al., 2000; Kim \& Im, 2018; Zeng et al., 2013). Of course, cabling and physical setup in the car do generate some extra, though negligible, work. As mentioned, our data analysis supports research on drivers' mental state, most notably cognitive load, fatigue, and drowsiness (Ahn et al., 2016; Borghini et al., 2014; Ingre et al., 2006; Lal \& Craig, 2001). Our setup is able to record potentially crucial changes in mental state, such as cognitive workload, and can thus be used to study such important research topics in situ.

\subsection{Case 3: Ashtanga Vinyasa Yoga practice in situ}

\subsubsection{Description of an Ashtanga Vinyasa Yoga practice}

A pilot experiment of an Ashtanga Vinyasa Yoga practice (ashtanga for short) was conducted to further test robustness and flexibility of the setup in studies with movement. Ashtanga is a moving yoga practice consisting of a standardized sequence of physical postures, connected by flowing movements and synchronized breathing patters, performed the same way every time (Mikkonen et al., 2008). Two participants practiced the half primary series by following instructions from a free online class (Ashtanga Yoga Full Primary Series with Ty Landrum, 2020) in their own living room. They were instructed to perform the practice as normal as possible. The sensors were attached to one participant, and fNIRS wires were secured to the participant's body by means of a tight-fitting top. The participant wearing the sensor cap did not perform postures that would affect electrode and optode scalp connection (e.g. headstand), and had two years of experience practicing other yoga types. Several sessions have been recorded. The illustrated session (Fig. 7) collected data from EEG, fNIRS, electrocardiography and two webcams simultaneously. However, only fNIRS data has been analyzed thus far and will discussed in the following section.
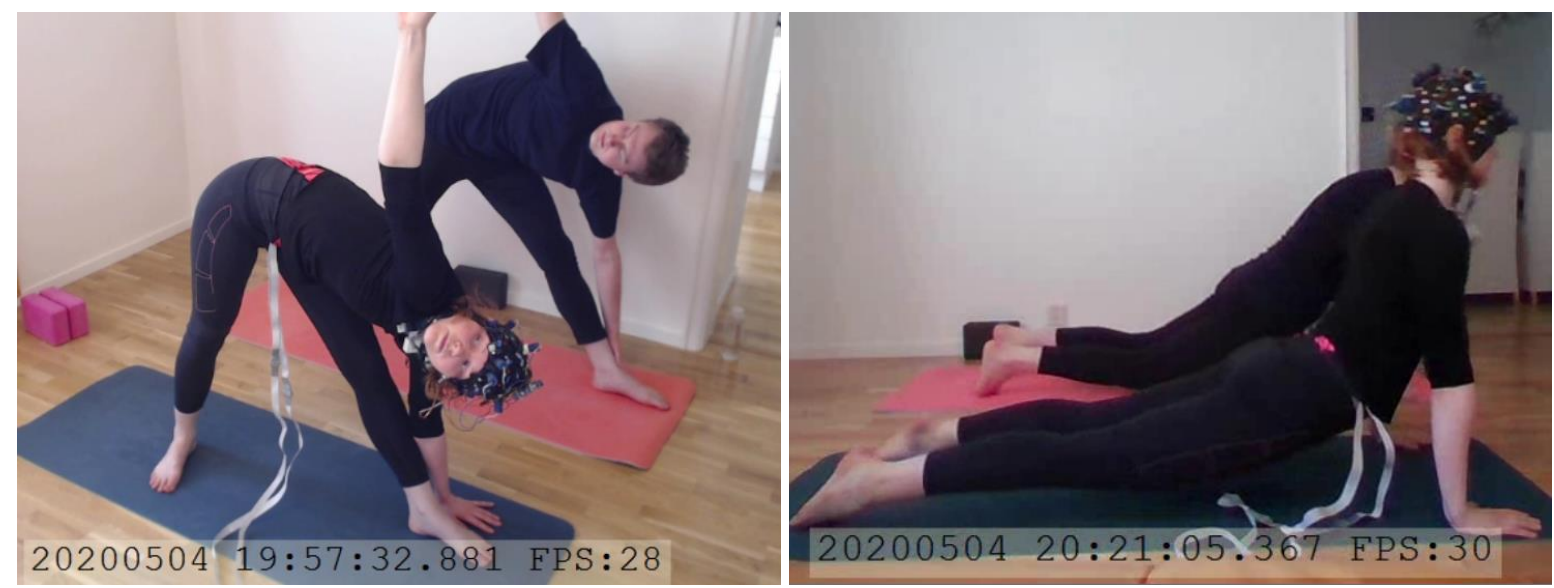

Figure 7. Screenshots from webcam recording during the ashtanga practice.

\subsubsection{Results from preliminary data analysis}

Result of preliminary analysis fNIRS. Fig. 8 depicts hemoglobin data, and Fig. 9 data after additional filtering. In Fig $8 \Delta \mathrm{HbO}_{2}$ and $\Delta \mathrm{HbR}$ are dominated by periodic large spikes across all channels. These are motion artifacts caused by the transition between postures. By investigating data from approx. $500-1000 \mathrm{~s}$, we see several "equal" periods where high quality data is collected. Combined with domain knowledge and video recording we infer that these "good" periods are collected during the posture downwards-facing dog. In between each good period there are similar motion artifacts, stemming from the transition between postures. Towards the end of the session the number of seated positions increase, and from $3500 \mathrm{~s}$ and out the participant performed less postures which resulted in less transitions and thus less artifacts as is visible in Fig. 8. By applying a TDDR 
filter (corrects motion artifacts by down-weighting outlier fluctuations) (Fishburn et al., 2019) we can view trend lines in the data over time. As we can see most channels follow the same trend with and same artifacts, being more dispersed at the beginning and end of the practice.

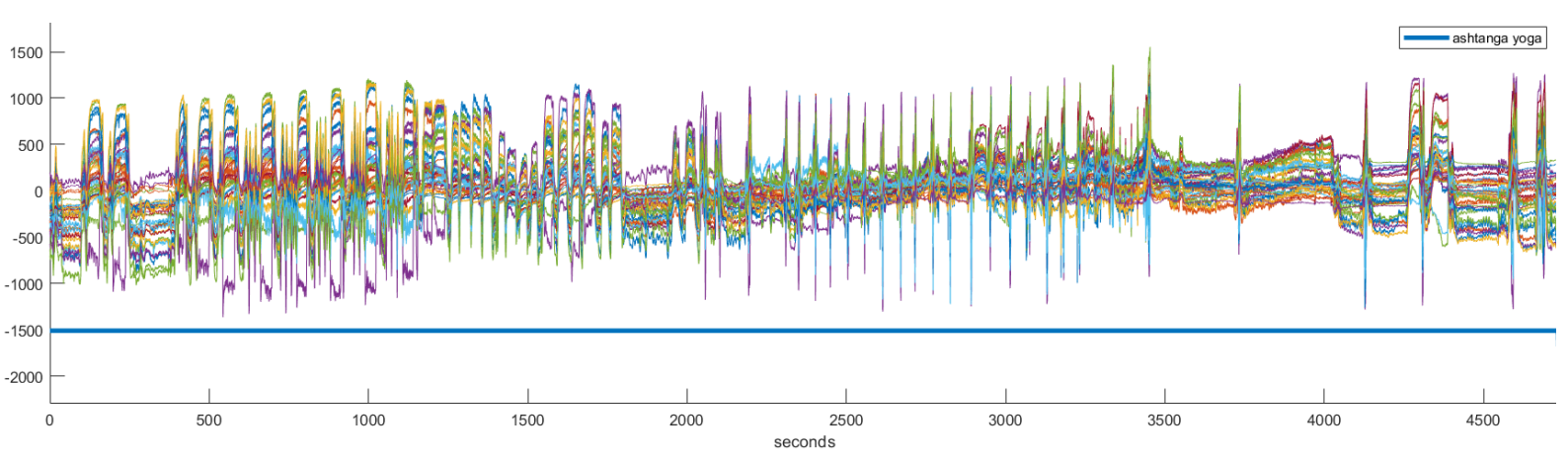

Figure 8. $\triangle \mathrm{HbO}_{2}$ and $\triangle \mathrm{HbR}$.

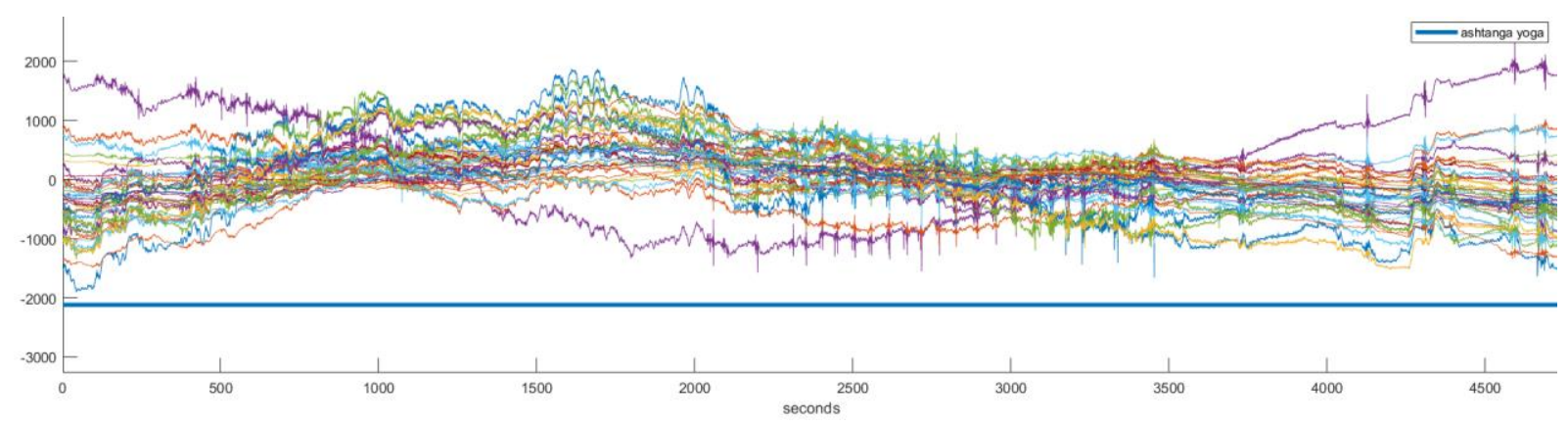

Figure 9. $\triangle \mathrm{HbO}_{2}$ and $\triangle \mathrm{HbR}$ data with TDDR filter (Fishburn et al., 2019). Continuous fNIRS data stream throughout moving sequence of $\sim 1 \mathrm{~h}$ and $18 \mathrm{~min}$.

\subsubsection{Lessons learned - motion artifacts are present; However, we can still acquire acceptable data}

In this yoga practice we experience movement artifacts in fNIRS data that are systemic and occur across all channels. Since the newer statistical models (AR-IRLS mentioned in section 2) account for this the systemic artifacts will not be particularly problematic in further statistical inference testing. This differs from signal artifacts discussed in section 3.1.3 and Fig. $4 \mathrm{~b}$ since the latter affects only one channel. Although not needed for statistical testing, the TDDR motion artifact filter enable visual investigation of overall trends in the data - to the extent that we can separate channels from each other. One channel (S1-D2, purple in Fig. 9) behaves differently from the others, making it subject for additional scrutiny. It's located toward the montage's back left. The EEG battery pack was head mounted, and its weight might alleviate pressure from optode tips, or cause loss of contact with the scalp. This contributes to the large spikes in the data. Furthermore, battery position was slightly to the left on the top of the head, making S1-D2 one of the closest channels. This may contribute to the deviating behavior of the channel. The battery pack should have been placed on the body instead of the head. To summarize, despite motion artifacts, the setup can be used to acquire reasonable data by using motion artifact correction algorithms.

\section{EXAMPLES OF POTENTIAL EXPERIMENTS IN DESIGN RESEARCH}

The increasingly complex experiment demonstrations showcase feasibility of acquiring neuroimaging data from participants who are 1) seated at a desk conducting tasks within desk range, 2) driving a vehicle (conducting a multifaceted task outside a laboratory), and 3) moving dynamically in space by exercising intentional motor control. We interchange these tasks for typical tasks in design research to gain understanding of potential experiments. Studies on sketching conventionally have participants seated at a desk while they use sketches as means to complete a design task. Therefore, it is possible to use the EEG+fNIRS setup to measure the cognitive processes that take place while sketching. This may even be coupled with the think aloud protocol for triangulation purposes, and to see if participant introspection concurs with neuroimaging data. The setup may also be used in product evaluation in 
which users wears EEG+fNIRS while interacting with the product to investigate whether the product is fulfilling its intended purpose, e.g. is a proposed interface as intuitive as intended? This can be particularly helpful when comparing several design alternatives. There are many opportunities to investigate the cognition of designers while engaged in the design process, as highlighted by Hay et al. (2020). Given that design is a collaborative activity often conducted by groups of individuals, it is interesting to investigate how they work together to reach mutual understanding and a design problem solution. The neurological synchrony of designers in ecologically valid situations may indeed be investigated with the proposed EEG+fNIRS setup, building upon prior research (Mayseless et al., 2019).

\section{CONCLUDING REMARKS}

This paper demonstrates a wearable experimental sensor setup collecting multimodal EEG+fNIRS neuroimaging data capture in situ. We demonstrate to which variety of experimental scenarios the setup can be appropriated to. Three distinct use cases were tested: a conventional human-computer interaction experiment, a driving experiment in situ involving city and highway driving, and a moving yoga practice in situ. Resulting data on cognitive load from highly ecologically valid experimental setups are presented. We also discuss lessons learned including signal quality, motion artifacts, and illustrate acceptable and unacceptable data artifacts. We propose to use this setup to measure construct such as cognitive load and suggest a variety of potential experiments pertinent to design research. Taken together, this demonstrate that an EEG+fNIRS sensor setup is both feasible and valuable for design research, and expands the range of what is possible within a design research experiment. We encourage the community to use the setup and adapt it to your in situ experiments.

\section{ACKNOWLEDGMENTS}

This research is supported by strategic funds from the department for Mechanical and Industrial Engineering (MTP) at NTNU.

\section{REFERENCES}

Ahn, S., \& Jun, S. C. (2017). Multi-Modal Integration of EEG-fNIRS for Brain-Computer Interfaces - Current Limitations and Future Directions. Frontiers in Human Neuroscience, 11. https://doi.org/10.3389/fnhum.2017.00503

Ahn, S., Nguyen, T., Jang, H., Kim, J. G., \& Jun, S. C. (2016). Exploring Neuro-Physiological Correlates of Drivers' Mental Fatigue Caused by Sleep Deprivation Using Simultaneous EEG, ECG, and fNIRS Data. Frontiers in Human Neuroscience, 10. https://doi.org/10.3389/fnhum.2016.00219

Antonenko, P., Paas, F., Grabner, R., \& van Gog, T. (2010). Using Electroencephalography to Measure Cognitive Load. Educational Psychology Review, 22(4), 425-438. https://doi.org/10.1007/s10648-0109130-y

Ashtanga Yoga Full Primary Series with Ty Landrum. (2020). https://www.youtube.com/watch?v=K$\mathrm{s} 4 \mathrm{IIxVBc} 8 \& \mathrm{t}=2607 \mathrm{~s}$

Balters, S., \& Steinert, M. (2017). Capturing emotion reactivity through physiology measurement as a foundation for affective engineering in engineering design science and engineering practices. Journal of Intelligent Manufacturing, 28(7), 1585-1607. https://doi.org/10.1007/s10845-015-1145-2

Barker, J. W., Aarabi, A., \& Huppert, T. J. (2013). Autoregressive model based algorithm for correcting motion and serially correlated errors in fNIRS. Biomedical Optics Express, 4(8), 1366. https://doi.org/10.1364/BOE.4.001366

Borghini, G., Astolfi, L., Vecchiato, G., Mattia, D., \& Babiloni, F. (2014). Measuring neurophysiological signals in aircraft pilots and car drivers for the assessment of mental workload, fatigue and drowsiness. Neuroscience \& Biobehavioral Reviews, 44, 58-75. https://doi.org/10.1016/j.neubiorev.2012.10.003

Cairns, P. E., \& Cox, A. L. (2008). Research methods for human-computer interaction. Cambridge University Press.

Cisler, D., Greenwood, P. M., Roberts, D. M., McKendrick, R., \& Baldwin, C. L. (2019). Comparing the Relative Strengths of EEG and Low-Cost Physiological Devices in Modeling Attention Allocation in Semiautonomous Vehicles. Frontiers in Human Neuroscience, 13. https://doi.org/10.3389/fnhum.2019.00109

Consolvo, S., Harrison, B., Smith, I., Chen, M. Y., Everitt, K., Froehlich, J., \& Landay, J. A. (2007). Conducting In Situ Evaluations for and With Ubiquitous Computing Technologies. International Journal of HumanComputer Interaction, 22(1-2), 103-118. https://doi.org/10.1080/10447310709336957 
Delorme, A., \& Makeig, S. (2004). EEGLAB: An open source toolbox for analysis of single-trial EEG dynamics including independent component analysis. Journal of Neuroscience Methods, 134(1), 9-21. https://doi.org/10.1016/j.jneumeth.2003.10.009

Dybvik, H., Erichsen, C. K., \& Steinert, M. (Under Review). Description of a Wearable Electroencephalography and Functional Near-Infrared Spectroscopy (EEG+fNIRS) for in Situ Experiments on Design Cognition. Proceedings of the Design Society: International Conference on Engineering Design.

Fishburn, F. A., Ludlum, R. S., Vaidya, C. J., \& Medvedev, A. V. (2019). Temporal Derivative Distribution Repair (TDDR): A motion correction method for fNIRS. NeuroImage, 184, 171-179. https://doi.org/10.1016/j.neuroimage.2018.09.025

Gero, J. S., \& Milovanovic, J. (2020). A framework for studying design thinking through measuring designers' minds, bodies and brains. Design Science, 6, e19. https://doi.org/10.1017/dsj.2020.15

Goucher-Lambert, K., Moss, J., \& Cagan, J. (2019). A neuroimaging investigation of design ideation with and without inspirational stimuli-Understanding the meaning of near and far stimuli. Design Studies, 60, 1-38. https://doi.org/10.1016/j.destud.2018.07.001

Hay, L., Cash, P., \& McKilligan, S. (2020). The future of design cognition analysis. Design Science, 6. https://doi.org/10.1017/dsj.2020.20

Herold, F., Wiegel, P., Scholkmann, F., \& Müller, N. G. (2018). Applications of Functional Near-Infrared Spectroscopy (fNIRS) Neuroimaging in Exercise-Cognition Science: A Systematic, Methodology-Focused Review. Journal of Clinical Medicine, 7(12), 466. https://doi.org/10.3390/jcm7120466

Ingre, M., Åkerstedt, T., Peters, B., Anund, A., \& Kecklund, G. (2006). Subjective sleepiness, simulated driving performance and blink duration: Examining individual differences. Journal of Sleep Research, 15(1), 4753. https://doi.org/10.1111/j.1365-2869.2006.00504.x

Jacko, J. A. (2012). The human-computer interaction handbook: Fundamentals, evolving technologies, and emerging applications (3rd ed.). CRC Press.

Jung, T.-P., Makeig, S., Humphries, C., Lee, T.-W., McKeown, M. J., Iragui, V., \& Sejnowski, T. J. (2000). Removing electroencephalographic artifacts by blind source separation. Psychophysiology, 37(2), $163-178$. https://doi.org/10.1111/1469-8986.3720163

Kim, D.-W., \& Im, C.-H. (2018). EEG Spectral Analysis. In C.-H. Im (Ed.), Computational EEG Analysis: Methods and Applications (pp. 35-53). Springer. https://doi.org/10.1007/978-981-13-0908-3_3

Klimesch, W. (1999). EEG alpha and theta oscillations reflect cognitive and memory performance: A review and analysis. Brain Research Reviews, 29(2), 169-195. https://doi.org/10.1016/S0165-0173(98)00056-3

Lal, S. K. L., \& Craig, A. (2001). A critical review of the psychophysiology of driver fatigue. Biological Psychology, 55(3), 173-194. https://doi.org/10.1016/S0301-0511(00)00085-5

Mayseless, N., Hawthorne, G., \& Reiss, A. L. (2019). Real-life creative problem solving in teams: FNIRS based hyperscanning study. NeuroImage, 203, 116161. https://doi.org/10.1016/j.neuroimage.2019.116161

Mikkonen, J., Pedersen, P., \& McCarthy, P. W. (2008). A Survey of Musculoskeletal Injury among Ashtanga Vinyasa Yoga Practitioners. International Journal of Yoga Therapy, 18(1), 59-64. https://doi.org/10.17761/ijyt.18.1.10748p25k2558v77

Okamoto, M., Dan, H., Shimizu, K., Takeo, K., Amita, T., Oda, I., Konishi, I., Sakamoto, K., Isobe, S., Suzuki, T., Kohyama, K., \& Dan, I. (2004). Multimodal assessment of cortical activation during apple peeling by NIRS and fMRI. NeuroImage, 21(4), 1275-1288. https://doi.org/10.1016/j.neuroimage.2003.12.003

Oostenveld, R., \& Praamstra, P. (2001). The five percent electrode system for high-resolution EEG and ERP measurements. Clinical Neurophysiology, 112(4), 713-719. https://doi.org/10.1016/S1388-2457(00)00527-7

Pinti, P., Tachtsidis, I., Hamilton, A., Hirsch, J., Aichelburg, C., Gilbert, S., \& Burgess, P. W. (2018). The present and future use of functional near-infrared spectroscopy (fNIRS) for cognitive neuroscience. Annals of the New York Academy of Sciences. http://dx.doi.org/10.1111/nyas.13948

Piper, S. K., Krueger, A., Koch, S. P., Mehnert, J., Habermehl, C., Steinbrink, J., Obrig, H., \& Schmitz, C. H. (2014). A Wearable Multi-Channel fNIRS System for Brain Imaging in Freely Moving Subjects. NeuroImage, 85(0 1). https://doi.org/10.1016/j.neuroimage.2013.06.062

Santosa, H., Zhai, X., Fishburn, F., \& Huppert, T. (2018). The NIRS Brain AnalyzIR Toolbox. Algorithms, 11(5), 73. https://doi.org/10.3390/a11050073

Steinert, M., \& Jablokow, K. (2013). Triangulating front end engineering design activities with physiology data and psychological preferences. 109-118.

Sterman, M., Mann, C., \& Kaiser, D. (1993). Quantitative EEG patterns of differential in-flight workload.

Zeng, H., Song, A., Yan, R., \& Qin, H. (2013). EOG Artifact Correction from EEG Recording Using Stationary Subspace Analysis and Empirical Mode Decomposition. Sensors (Basel, Switzerland), 13(11), 14839_ 14859. https://doi.org/10.3390/s131114839 